\title{
Burden Assessment of Thyroid cancer in Iran from 1990 to 2010: Lessons Obtained from Global Burden of Disease Report 2010
}

\author{
Mitra Modirian ${ }^{1 *}$, Zahra Cheraghi', ${ }^{2,1}$ Shadi Rahimzadeh ${ }^{3,1}$, Sahar Saeedi \\ Moghaddam $^{4,1}$, Alireza Mosavi Jarrahi ${ }^{3}$
}

\begin{abstract}
Background: Thyroid tumors are generally regarded as rare malignancies. Nowadays, however, their global incidence is growing continuously partially due to western life style and utilization of more sensitive methods of early detection. It is approximately three times more prevalent in females than in males. Most cases of thyroid cancer are asymptomatic nodules or just have local cervical symptoms or adenopathy in early stages. Materials and Methods: The Global Burden of Diseases report 2010 study (released 3/2013) profited from 100 collaborators worldwide and used a vast network of data on health outcomes, vital registries, and population surveys. It shared many of the Global Burden of Diseases 1990 principal databases such as all available data on injuries, diseases, risk factors, as well as comparable metrics, and used different scientific approved methods to estimate important health status data like: death rate, life expectancy, healthy adjusted life expectancy, disability-adjusted life years (DALY), years of living lost due to premature death and years of life with disabilities. Results: DALY as thyroid cancer burden per 100,000 Iranian populations had increased by about 14\% during 1990 to 2010 in all ages; from 6.1 (95\% UI 4.2-9.74) years in 1990 to 6.95 (95\% UI 5.06-9.18) years in 2010 in both sex. The 2010 peak age-group was estimated at 45-49 years in males and 40-45 years in females.
\end{abstract}

Keywords: Thyroid - cancer - burden - Iran - DALY

Asian Pac J Cancer Prev, 16 (17), 7743-7748

\section{Introduction}

Thyroid cancer is, globally, the most common cancer of endocrine system that affects young adults with a maximum age of about 45 years (Stewart et al., 2003). It comprises a group of tumors with remarkably various features and different prognosis; it is approximately three times more prevalent in female than in male. Most cases of thyroid cancer are demonstrated as thyroid asymptomatic nodules or just have local cervical symptoms or adenopathy in early stages. Some forms of thyroid cancer like anaplastic types are untreatable, meanwhile early diagnosis and treatment are effective for most types and result in 10-year survival rates of $92-98 \%$, depending on the type (Cecil et al., 2012). The most known risk factors for this malignancy are genetic susceptibility and Ionizing radiation, but some probable factors have also been studied like benign thyroid condition, hormonal and reproductive factors, iodine intake, height and body mass index (Maso et al., 2009; Kitahara et al., 2014; Xhaard et al., 2014).

Although thyroid tumor has already been introduced as a rare malignancy, nowadays its global incidence is growing continuously partially due to western life style and utilization of more sensitive methods of early detection (Stewart et al., 2003; Sankaranarayanan et al., 2014). Based on Globocan report, new cases of thyroid cancer was 212,000 with 35,000 death in 2008, while it became 298,102 new case with 39,769 death in 2012, which showed an increase of more than $40 \%$ and $13 \%$, respectively, in incidence and mortality rates worldwide; in addition it has been predicted that its incidence will increase to 398,436 cases and mortality rate will reach 64,485 deaths in 2030 , worldwide.

Also Thyroid cancer has been introduced as the fastest increasing cancer in both male and female in the US after mid-1990s with 10-years total cost of $\$ 18$ to $\$ 21$ billion dollars from 2010-2019 (Sundram et al., 2006; Aschebrook-Kilfoy et al., 2013). Another cost analysis study described that late detection of thyroid cancers encompasses 1.8-2.5 folds more medical costs than early detected cases: total medical costs of $\$ 4,936$ in localized stage compared to $\$ 11,409$ for metastatic ones (Shin et al., 2012). According to a study on burden of cancers in Korea 2000-2020,thyroid cancer together with colon and

${ }^{I}$ Non-communicable Diseases Research Center, Endocrinology \& Metabolism Population Sciences Institute, ${ }^{2}$ Department of Epidemiology and Biostatistics, Tehran University of Medical Sciences, ${ }^{3}$ Department of Epidemiology, ${ }^{4}$ Department of Biostatistics, Faculty of Paramedical Sciences, Shahid Beheshti University of Medical Sciences, Tehran, Iran *For correspondence: dr.mitramodirian@gmail.com 
breast cancers had the highest levels of disability adjusted life years (DALY), and had the first rank of incidence and fifth of DALY in young female aged 20-39 years old in 2020 (Park et al., 2013). Figure-1 shows the Globocan estimates and projection of thyroid cancer incidence and mortality trend from 1990 to 2035 (Parkin et al., 1999; Pisani et al., 1999; Parkin et al., 2001; Parkin et al., 2005; Ferlay et al., 2010; Jemal et al., 2011).

According to a study on old population in Iran 2001-2005, the incidence rate of thyroid cancer was estimated 16 and 29.2 per 100,000 male and female, respectively (Akbari et al., 2011). Another local study in Tehran metropolis 1998-2001, showed that female aged 75 years and more, have the most annual incidence rate of thyroid cancer $(8.7$ per 100,000 female in contrast to 5.4 per 100,000 male in the same age group annually) (Mohagheghi et al., 2009).

The report of Institute for Health Metrics and Evaluation (IHME), Global Burden of Disease (GBD), in 2010 (released 3/2013) has revealed that all ages' DALYs of thyroid cancer in North Africa and Middle East increased by $18 \%$, from 10.4 (95\% UI 7.33-13.65) years per 100,000 in 1990 to 12.3 (95\% UI 8.49-15.46) years per 100,000 in 2010 , also $11 \%$ increasing in Global DALYs of thyroid cancer in the same time (Murray et al., 2013). Despite the sharp increase in thyroid cancer prevalence, its potential burden and economic challenge have not been well studied; hence, because of different reasons including the rapid increase in the incidence of disease, the prevalence of disease among youth, and the number of affected people who need long term care, as a first step to control the cancer it is essential to determine the burden of thyroid cancer. On the other hand, there is no published scientific document on thyroid malignancies burden estimation among Iranian male and female (the last national study of national burden of diseases in Iran was performed in 2003 but it did not include thyroid cancer burden). So we decided to address the burden of thyroid cancer in Iranian population during 1990 to 2010 based on IHME Global report 2010.

\section{Materials and Methods}

\section{Assessing health outcomes:}

Death rates (Murray and Lopez, 2013) were estimated using vital registry datasets. To use distribution methods, vital registry data from more than 100 countries was collected. The Gaussian process regression was used to generate the final mortality estimates about under and over 5-year age groups for about 187 countries (the countries with a population of more than 50,000 individuals in the year 2000).

Disability Weight measurement (Jung et al., 2013) was conducted using two different surveys. One was household survey of adults aged 18 years or older (it was conducted either face to face or via telephone interview) in 20092010. Another survey was an open-access and web-based survey, which was carried out in 2010-2011. Both surveys included comparative questions in two hypothetical fields with different and randomly selected health states regarding the healthier individual status. The web based survey had some extra questions addressing overall health benefits of different life saving or preventive programs.

The disability weights for about 220 health conditions were estimated. Considering cancer, the least mean disability weights, with 95\% Uncertainty Intervals (UI), were estimated for Mastectomy 0.038 (95\% UI 0.0220.059 ) and Stoma 0.086 (95\% UI 0.055-0.131). The results were in contrast with the estimated major weights, that is: Terminal phase of disease with medication 0.508 (95\% UI 0.348-0.670) or without it 0.519 (95\% UI 0.356-0.683). Cancer diagnosis and primary therapy with disability weight estimations of 0.294 (95\% UI 0.199-0.411) and metastatic cancer with 0.484 (95\% UI 0.330-0.643) were placed between the two borderlines.

Healthy-adjusted Life Expectancy (HALE) (Salomon et al., 2013) was assessed for two points in time (1990 and 2010), age-specific mortality rates and mentioning 1160 sequel with related disability weight of 220 health status. In order to capture co-morbidities in individuals, Mont Carlo simulation method was used. For age-specific mortality, life table calculation was used based on two estimations: mortality rates between age groups of 0-5 and 14-60 years and translation of these measures into a set of age-specific mortality rates. HALE estimations were produced through the conventional life table using the Sullivan method for each population by sex, country, and year.

Years Lived with Disability (YLD) (Vos et al., 2013) was computed for just 289 of 291 conditions which were non-fatal causes of disabilities. To analyze prevalence, incidence, remission, duration, and mortality a systematic review analysis (Bayesian meta-regression method, DisMod-MR) was performed on many different sources like population diseases registries, published studies, surveys, cohort studies, and hospital data. YLDs by sex, country, year level, and cause at age were adjusted via simulation methods to calculate co morbidity. The prevalence of four cancer sequels (diagnosis or treatment phase, recurrence, remissions, and terminal phase) and the natural history of cases were estimated through calculating five-year survival and relative duration of each cancer phase.

Years of Life Lost due to premature death (YLL) (Lozano et al., 2013) was estimated based on the difference between LE (Life Expectancy) and the date of cause specific mortality. Like other major causes of death, CODEm was used for estimating cancer YLL through three steps: developing a range of plausible statistical models for each cause, performing all component models and ensembles, and selecting the best performing model based on out-of-sample predicting validity.

Disability Adjusted Life Years (DALYs) (Murray et al., 2013) was calculated as the sum of the years of life lost (YLL) and years lived with disability (YLD) for 291 diseases and injuries, 20 age groups, both sexes, in 187 countries, for five points in time from 1990 to 2010 . YLL and YLD were not age weighted, nor were they discounted.

The GBD 2010 study benefited from 100 collaborators worldwide and used a vast network of data on health outcomes, vital registries, and population surveys. The 
GBD report 2010 (released 3/2013) shares many of the GBD 1990 principal databases such as all available data on injuries, diseases, risk factors, as well as comparable metrics.

\section{Results}

As it has been shown in Table-1, Thyroid cancer burden (DALYs) per 100,000 Iranian populations had increased by about $14 \%$ during 1990 and 2010 in all ages (from 6.1 (95\% UI 4.2-9.74) years in 1990 to $6.95(95 \%$ UI 5.06-9.18) years in 2010 in both sex).

GBD report-2010 has revealed that the prevalence rate of thyroid cancer in Iranian population by sex and age-groups increased from 2591 (95\% UI 1653-3941.1) persons in 1990 to 6126 (95\% UI 4038.7-8624.9) persons in 2010 in both sexes, from 783.8 (95\% UI 458.62-1409.43 ) in 1990 to 2001 (95\% UI 1150.04-3132.1) persons in 2010 in males, and from 1807 (95\% UI 989.94-3061.14) in 1990 to 4126 (95\% UI 2317.52-6444.91) persons in 2010 in females. The 2010 peak age-group was estimated 45-49 years in males (mean prevalence of 267.72 with $95 \%$ UI 72.28-725.37) and 40-45 years in females (mean prevalence 697.61 with 95\% UI 228.98-1620.96) (Figure-2).

The trend of mortality per 100,000 Iranian male and female shows that in 1990 thyroid cancer in all Iranian population caused 103.106 (95\% UI 72.94-152.72) deaths which increased to 193.513 (95\% UI 139.41-255.88) deaths in 2010. It shows an increase in mortality rate which had no significant difference between different agegroups from 1990 to 2010; however there was a decrease in DALYs in all age groups during the same period of time (Figures $3 \& 4$ ).
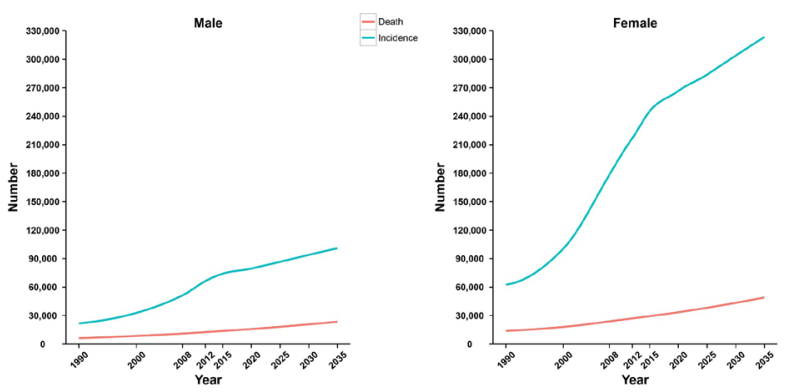

Figure 1. Number of Death and Incidence of thyroid cancer from 1990 to 2035 in male and female

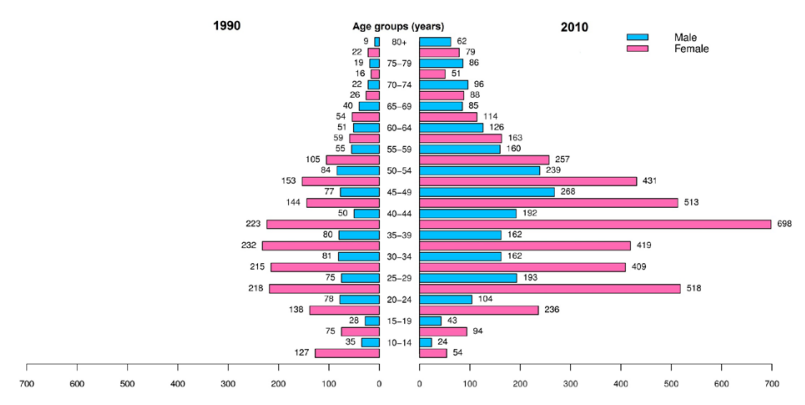

Figure 2. Prevalence of Thyroid Cancer by age Groups, and sex in 1990 and 2010

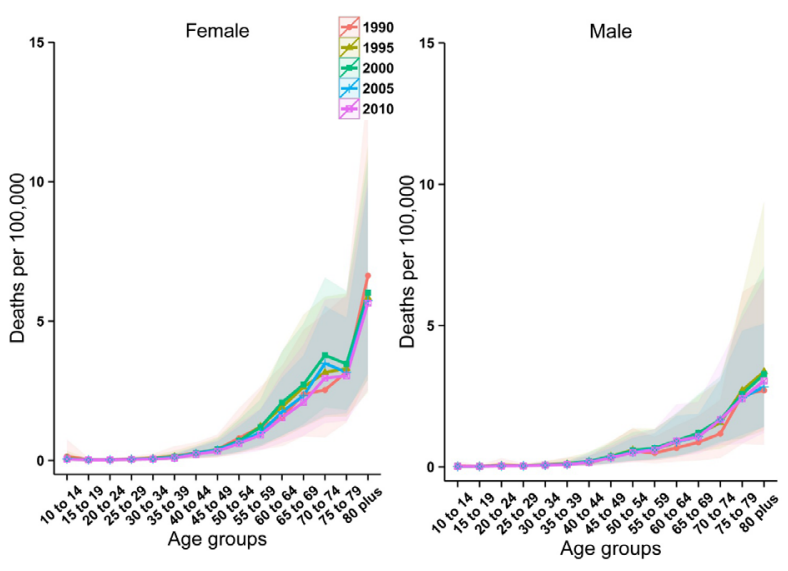

Figure 3. Deaths per 100,000 of Thyroid Cancer by age Groups from 1990 to 2010 in Female and Male

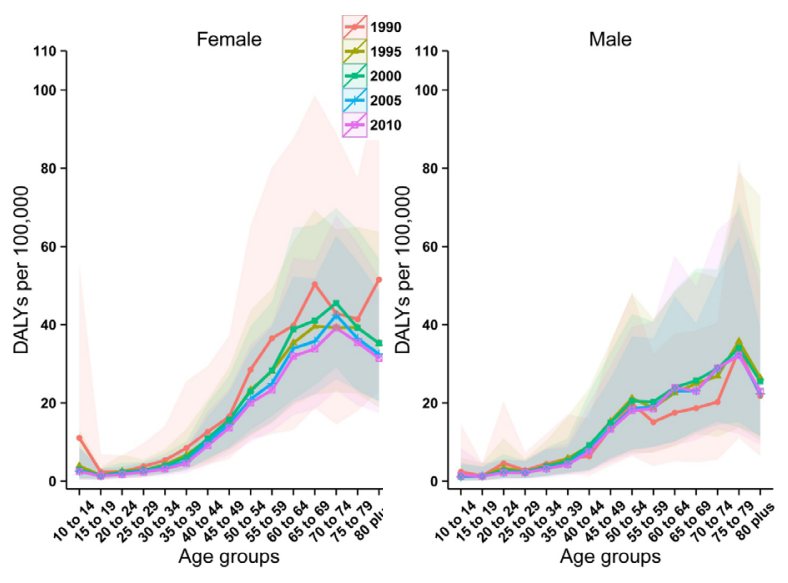

Figure 4. DALYs per 100,000 of thyroid cancer by age groups from 1990 to 2010 in female and male

Table 1. Thyroid Cancer Burden in Iranian Population: 1990 and 2010

\begin{tabular}{|c|c|c|c|c|c|c|}
\hline $\begin{array}{l}\text { Gender } \\
\text { Estimation }\end{array}$ & DALY & $\begin{array}{l}1990 \\
\text { YLL }\end{array}$ & YLD & DALY & $\begin{array}{l}2010 \\
\text { YLL }\end{array}$ & YLD \\
\hline \multicolumn{7}{|l|}{ Male } \\
\hline Rate per 100,000 & 4.27 & 4.15 & 0.12 & 5.88 & 5.66 & 0.22 \\
\hline Uncertainty Interval & $2.6642-7.35771$ & $2.54761-7.28467$ & $0.0318109-0.294932$ & $3.54681-8.61002$ & $3.37892-8.43101$ & $0.0703715-0.0703715$ \\
\hline \multicolumn{7}{|l|}{ Female } \\
\hline Rate per 100,000 & 7.96 & 7.7 & 0.26 & 8.06 & 7.62 & 0.44 \\
\hline Uncertainty Interval & $4.74023-14.6524$ & $4.42761-14.4226$ & $0.084233-0.594981$ & $4.94016-11.6927$ & $4.46093-11.172$ & $0.14921-0.913356$ \\
\hline \multicolumn{7}{|l|}{ Both } \\
\hline Rate per 100,000 & 6.1 & 5.91 & 0.19 & 6.95 & 6.63 & 0.33 \\
\hline Uncertainty Interval & $4.20782-9.74449$ & $3.9736-9.52726$ & $0.0746717-0.385846$ & $5.0646-9.18383$ & $4.7649-8.83707$ & $0.142129-0.602457$ \\
\hline
\end{tabular}




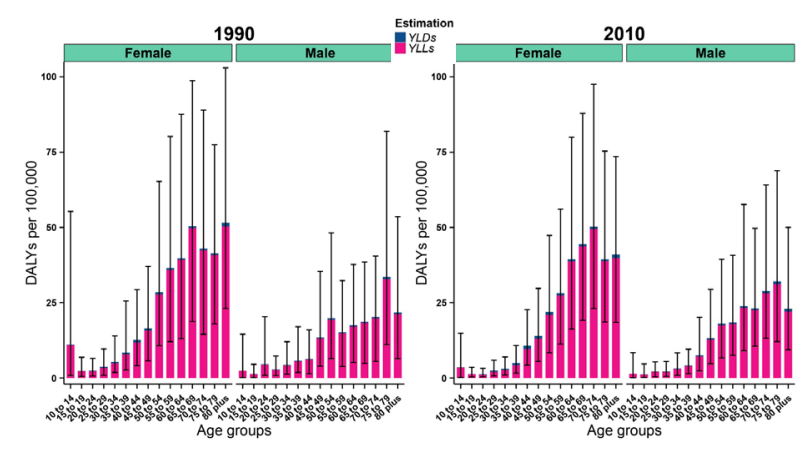

Figure 5. DALYs per 100,000 by age Groups, sex, YLLs, and YLDs in 1990 and 2010

The comparison of thyroid cancer YLL and YLD in Iranian male and female between 1990 and 2010 is presented in Figure-5. As expected, DALYs in females are obviously higher than in male in all age-groups (except 20-35 years). The highest DALYs in male was observed in age-group 75-79 years with 32.12 (95\% UI 12.1-68.81) DALYs per 100,000 in both years, but in female the highest level of DALYs among females' age-groups was different: 51.57 (95\% UI 23.07-102.95) years in 1990 in female aged 80+ years old and 50.18 (95\% UI 23.0797.54) years per 100,000 in 2010 in female aged $70-74$ years old. The highest levels of YLL and YLD per 100,000 in 2010 were estimated as 31.18 (95\% UI 10.63-68.05) years and 0.94 (95\% UI 0-4.01) year both in male aged group 75-79 and in female as 49.46 (95\% UI 22.48-96.65) years in age group 70-74 and 1.17 (95\% UI 0.09-3.88) years in age group 40-44 respectively.

\section{Discussion}

In the last three decades, thyroid malignancy incidence has obviously raised up, and it became three fold more prevalent in female than in male worldwide (except Sweden, Norway and Spain (Kilfoy et al., 2009)), however there was difference in incidence rates which were due to differences in geographical locations and environmental factors (Pellegriti et al., 2013). Despite the sharp rise in thyroid cancer prevalence, its potential burden and economic challenge has not been well studied.

The international reports showed average increasing about $48 \%$ among males and $66.7 \%$ among females during the past several decades, also the age-adjusted international thyroid malignancy rates from 1998-2002 increased up to 5-fold for men and 10-fold for women based on geographic region (Kilfoy et al., 2009). In the Asia-pacific region, thyroid cancer is among the ten most prevalent cancers, despite of various patterns of health care systems, economies, cultures, ethnics and racial populations, also strategies for early diagnostic and initial treatments (Sundram et al., 2006). For example in Korea, the incidence rate of thyroid cancer has been increased from 6.3 in 1999 to 52.7 in 2010 with annual $24.2 \%$ increase (using age standardized incidence data) (Jung et al., 2013).

At the regional level, thyroid cancer is the fifth common cancer in Gulf Cooperation Council (GCC) and the second common cancer (after breast cancer) among female population of Arab countries (Hussain et al., 2013; Moore, 2013). Thyroid cancer was the second most common malignancy among female in Kingdom of Saudi Arabia in 2008; it was the cause of $8.8 \%$ of all malignancies and $12 \%$ of all female malignant cancers, based on different geographical locations between 2000 and 2010 (Ferlay et al., 2010). Also an eight-year followup during 1998-2006 in Oman showed that, considering the incidence rate, thyroid cancer was the second common cancer in female with 5.4/100,000 cases (Murray et al., 2013), while in Jordan 1996-2009, thyroid malignancies were ranked as the forth common cancer in adult females with age-standardized incidence rate of 4.9 per 100,000 population in 1996-2009 (Ismail et al., 2013).

According to a nationwide study in Turkey in 2010, thyroid cancer was estimated as the forth common cancer with 8.8 cases per 100,000 female (Moore, 2013); also it was reported as the forth common cancer in Yemen in females with 1.6 cases per 100,000 in 2000-2006 and with the highest incidence in age group of 60-64 years (Saleem et al., 2010). In United Arab Emirates, 2008 ,thyroid cancer was reported as the second common cancer in females (Loney et al., 2013). In addition Thyroid cancer increased by $141 \%$ in female population of South Korea from 1993 to 2002; it was the most common type of cancer in female in South Korea since 2003 and now it is one of the most common cancers in male with an increase of more than 24\% annually in both sexes (Jung et al., 2013; Moore, 2013). Also according to a national report of China during 2003-2007, the age-standardized incidence rate of thyroid cancer was calculated as 3.31 per 100,000 people with a mortality rate of 0.29 per 100,000 people (3.38 times higher in females than males); its incidence and mortality increased annually by $14.51 \%$ and $1.42 \%$, respectively (Liu et al., 2012).

The increase in thyroid cancer incidence is attributed to different causes like improved methods and techniques of detection and screening, being exposed to more risk factors, and the incidence of more cases of true cancer. Since the diagnostic equipments are unavailable and expensive in developing countries, and also as large thyroid nodules are observed in later stages, it can be said that the increasing trend of incidence could not be merely due to the utilization of sensitive diagnostic methods (Wartofsky, 2010; Pellegriti et al., 2013; Davies and Welch, 2014). For example in kingdom of Saudi Arabia such an increase probably is not merely due to increased detection and diagnosis but rather it is due to the real occurrence of this malignancy; besides, the improved detection methods could not obviously explain the 4 fold growing incidence in Czech patients from 1980s (Hussain et al., 2013; Lukas et al., 2013).

According to Globocan projection the incidence of thyroid cancer in Iran will increase by $35 \%$ from 2012 to 2025 , which would be equal in male and female, however, its mortality rate will increase by $66 \%$ from 610 to 1017 cases (more than $77 \%$ in female and $44 \%$ in male). GBD 2010 report also showed an increase of more than $136 \%$ in thyroid cancer prevalence in all ages in Iranian population, which was more than $155 \%$ in male and more than $128 \%$ 
in female during 1990 to 2010.

The high risk age-groups in different countries are not the same. Worldwide high risk age-group are about 40-45 years (Akbari et al., 2011). Although according to the last national cancer registry report in Iran 2009, the high risk age group for thyroid cancer was estimated as $80-84$ years with age specific incidence rate of $8.73(85+$ in male with incidence rate of 5.91 and 80-84 years in female with incidence rate of 12.50), in GBD 2010 report the highest prevalence was reported as 40-44 years among Iranian people, which is similar to the world range. In Czech population it was revealed that female aged 60 to 64 years old had the highest risk of thyroid cancer with median age at diagnosis of 55 and 57 years in female and male, respectively (Lukas et al., 2013).

In Yemen (2002-2006) the high risk groups of thyroid cancer were estimated as 60-64 years for male and 55-59 years for females (Saleem et al., 2010), while in Saudi Arabia high risk group for thyroid cancer were found in more younger age group of 35-45 years with median age of thyroid cancer occurrence of 40 and 44 years among females and males, respectively (Hussain et al., 2013). Moreover, the peak year of thyroid cancer in Omani female was similarly reported as 40-49 years (Nooyi and Al-Lawati, 2011). Nevertheless, this malignancy appears in both young and old adults (15-64 years old) in South Korea (Jung et al., 2010; Jung et al., 2013).

Thyroid cancer mortality rate in Iran was increased by more than $87 \%$ during 1990 to 2010 (from 0.03 in 1990 to $1.5 \%$ in 2010 among all death cause); the increase was more significant in male (123\%) than female (67\%). A review of GLOBOCAN projection for Iran 2025 also shows an increase of $66.7 \%$ in thyroid cancer mortality rate (44\% in male and 77\% in female) compared to 2012.

Despite a decrease of $0.94 \%$ in Global burden of thyroid cancer from 1990 to 2010, it shows more than $27 \%$ and $33 \%$ increase in developed and developing countries respectively. Also the regional estimation in central Asia shows near to $8 \%$ increasing. This change is different between countries; for example in Iran it had a moderate increase of $19.05 \%$, from 6.1 (4.2-9.74) years in 1990 to 6.95 (5.06-9.18) years in 2010 per 100,000 person in both sexes, which was similar to Yemen 12\% , Iraq 23\% ,Afghanistan $11 \%$ and Oman 24\% (despite highly growth in Bahrain 129.5\% and Pakistan 56.3\%).

In other regional countries, in contrary, thyroid cancer DALYs had a decrease like Egypt 22.67\%, Lebanon 2.5\%, Qatar 19.74\%, Saudi Arabia 31.53\%, United Arab Emirates 3.96\%, and Turkey 2.7\% (Murray et al., 2013). It is estimated that the highest DALYs in 2010, compared with 1990, is observed in Iranian male aged 75 to 79 years old and in younger female aged 70 to 74 years old (because of more early death in this age-group); while, the highest YLL in 1990 was observed in female aged 80 and more. Probably, such increase and decrease are mainly due to differences in applying early detection methods and equipment, exposure to risk factors, and also differences in mortality rates, rather than screening programs.

Thyroid malignancies have no obvious symptoms in early stages and the most common sign is "a cold nodule" without any hormonal activity. There are different studies about lifestyle risk factors for this type of cancer such as medical radiation exposure, nitrate, iodine intake, obesity and low physical activity which can affect the incidence of cancer (Stewart et al., 2003; Pellegriti et al., 2013; Schmid et al., 2013). Even though all causes of this type of malignancy are unknown, the increase in disease burden can be attributed to numerous different factors like: increased prevalence and low mortality rates, less exposing to probably known risk factors, simple early detection methods, new pharmacies and treatment methods, changes in the life style, detecting familial cases with genetic basis, and good prognosis and also high survival rates (Brown et al., 2011). So in near future it is unavoidable for healthcare system to face a huge number of thyroid cancer cases and cost burden. NASBOD 2013 study (National And Subnational study of Burden Of Diseases) which is the first comprehensive study of diseases burden and risk factors, through focusing on high incident and low mortality cancers can help health policy makers at national and provincial levels to pay more attention to cancer control programs (Kilfoy et al., 2009).

Conclusion:Improving diagnostic methods and treatments can convert the cancers from fatal diseases to chronic conditions which lead to increased cost burden in health care system. It is of great importance and value, especially in developing countries, to pay more attention to scientific research on thyroid cancer as an example of rare malignancies but with sharply growing incidence, and to consider different epidemiologic patterns, undefined life style risk factors and undetermined socio-economic burden in making health policies and planning cancer control programs. However, for epidemiologic studying of such diseases with raising incidence and low mortality, it seems better to study the burden via single measure of cancer burden (SMCB) which ranks cancers through both incidence and mortality equally, while DALY (Disability Adjusted Life Year) and other economic studies focus more on premature death (Cho et al., 2012).

\section{Acknowledgements}

The study is granted by Ministry of Health and Medical Education and Setad-e-Ejraie Farmane Imam. Also we would like to appreciate Institute of Health Metrics and Evaluation for data, also Dr. Masoud Moradi for his precise editing of the text.

\section{References}

Akbari ME, Rafiee M, Khoei MA, et al (2011). Incidence and survival of cancers in the elderly population in Iran: 20012005. Asian Pac J Cancer Prev, 12, 3035-9.

Aschebrook-Kilfoy B, Schechter RB, Shih Y-CT, et al (2013). The clinical and economic burden of a sustained increase in thyroid cancer incidence. Cancer Epidemiol Biomarkers Prev.

Brown RL, de Souza JA, Cohen E (2011). Thyroid cancer: burden of illness and management of disease. J Cancer, 2, 193-9.

Cecil RLF, Goldman L, Schafer AI 2012. Goldman's Cecil Medicine, Elsevier/Saunders.

Cho K-H, Park S, Lee K-S, et al (2012). A single measure of 


\section{Mitra Modirian et al}

cancer burden in Korea from 1999 to 2010. Asian Pacific J Cancer Prev, 14, 5249-55.

Dal Maso L, Bosetti C, La Vecchia C, et al (2009). Risk factors for thyroid cancer: an epidemiological review focused on nutritional factors. Cancer Causes Control, 20, 75-86.

Davies L, Welch HG (2014). Current Thyroid Cancer Trends in the United States. JAMA Otolaryngol-Head Neck Surgery.

Ferlay J, Shin HR, Bray F, et al (2010). Estimates of worldwide burden of cancer in 2008: GLOBOCAN 2008. Int J Cancer, 127, 2893-917.

Hussain F, Iqbal S, Mehmood A, et al (2013). Incidence of thyroid cancer in the Kingdom of Saudi Arabia, 2000-2011. Hematology/oncology and stem cell therapy.

Ismail SI, Soubani M, Nimri JM, et al (2013). Cancer incidence in Jordan from 1996 to 2009-a comprehensive study. Asian Pac J Cancer Prev, 14, 3527-34.

Jemal A, Bray F, Center MM, et al (2011). Global cancer statistics. CA Cancer J Clin, 61, 69-90.

Jung KW, Park S, Kong HJ, et al (2010). Cancer statistics in Korea: incidence, mortality and survival in 2006-2007. $J$ Korean Med Sci, 25, 1113-21.

Jung K-W, Won Y-J, Kong H-J, et al (2013). Cancer statistics in Korea: incidence, mortality, survival and prevalence in 2010. Cancer Res Treatmt, 45, 1-14.

Kilfoy BA, Zheng T, Holford TR, et al (2009). International patterns and trends in thyroid cancer incidence, 1973-2002. Cancer Causes Control, 20, 525-31.

Kitahara CM, Gamborg M, de González AB, et al (2014). Childhood height and body mass index were associated with risk of adult thyroid cancer in a large cohort study. Cancer Res, 74, 235-42.

Liu Y, Zhang S, Chen W, et al (2012). [Trend of incidence and mortality on thyroid cancer in China during 2003-2007]. Zhonghua liu xing bing xue za zhi Zhonghua liuxingbingxue zazhi, 33, 1044-8.

Loney T, Aw TC, Handysides DG, et al (2013). An analysis of the health status of the United Arab Emirates: the 'Big 4'public health issues. Global health action, $\mathbf{6}$.

Lozano R, Naghavi M, Foreman K, et al (2013). Global and regional mortality from 235 causes of death for 20 age groups in 1990 and 2010: a systematic analysis for the Global Burden of Disease Study 2010. Lancet, 380, 2095-128.

Lukas J, Drabek J, Lukas D, et al (2013). The epidemiology of thyroid cancer in the Czech Republic in comparison with other countries. Biomed Pap Med Fac Univ Palacky Olomouc Czech Repub, 157, 266-75.

Mohagheghi M-A, Mosavi-Jarrahi A, Malekzadeh R, et al (2009). Cancer incidence in tehran metropolis: the first report from the tehran population-based cancer registry. Arch Iran Med, 12, 15-23.

Moore MA (2013). Overview of cancer registration research in the Asian Pacific from 2008-2013. Asian Pac J Cancer Prev, 14, 4461-84.

Murray CJ, Lopez AD (2013). Measuring the global burden of disease. New England J Med, 369, 448-57.

Murray CJ, Vos T, Lozano R, et al (2013). Disability-adjusted life years (DALYs) for 291 diseases and injuries in 21 regions, 1990-2010: a systematic analysis for the Global Burden of Disease Study 2010. Lancet, 380, 2197-223.

Nooyi SC, Al-Lawati J (2011). Cancer incidence in Oman, 19982006. Asian Pac J Cancer Prev, 12, 1735-80.

Park JH, Lee KS, Choi KS (2013). Burden of cancer in Korea during 2000-2020. Cancer epidemiol, 37, 353-9.

Parkin DM, Bray F, Ferlay J, et al (2001). Estimating the world cancer burden: Globocan 2000. International J Cancer, 94, 153-6.

Parkin DM, Bray F, Ferlay J, et al (2005). Global cancer statistics,
2002. CA: a cancer journal for clinicians, 55, 74-108.

Parkin DM, Pisani P, Ferlay J (1999). Estimates of the worldwide incidence of 25 major cancers in 1990. International $J$ Cancer, 80, 827-41.

Pellegriti G, Frasca F, Regalbuto C, et al (2013). Worldwide increasing incidence of thyroid cancer: update on epidemiology and risk factors. J Cancer Epidemiol, 2013.

Pisani P, Parkin DM, Bray F, et al (1999). Estimates of the worldwide mortality from 25 cancers in 1990. IntJ Cancer, 83, 18-29.

Saleem HOB, Bawazir AA, Moore M, et al (2010). Five years cancer incidence in Aden Cancer Registry, Yemen. Asian Pac J Cancer Prev, 11, 507-11.

Salomon JA, Wang H, Freeman MK, et al (2013). Healthy life expectancy for 187 countries, 1990-2010: a systematic analysis for the global burden disease study 2010. Lancet, 380, 2144-62.

Sankaranarayanan R, Ramadas K, Qiao Y-l (2014). Managing the changing burden of cancer in Asia. BMC medicine, $\mathbf{1 2}, 3$.

Schmid D, Behrens G, Jochem C, et al (2013). Physical activity, diabetes, and risk of thyroid cancer: a systematic review and meta-analysis. Eur J Epidemiol, 28, 945-58.

Shin J-Y, Kim SY, Lee KS, et al (2012). Costs during the first five years following cancer diagnosis in Korea. Asian Pac J Cancer Prev, 13, 3767-72.

Stewart BW, Kleihues P, Organization WH, et al 2003. World cancer report, Iarc.

Sundram F, Robinson BG, Kung A, et al (2006). Welldifferentiated epithelial thyroid cancer management in the Asia Pacific region: a report and clinical practice guideline. Thyroid, 16, 461-9.

Vos T, Flaxman AD, Naghavi M, et al (2013). Years lived with disability (YLDs) for 1160 sequelae of 289 diseases and injuries 1990-2010: a systematic analysis for the Global Burden of Disease Study 2010. Lancet, 380, 2163-96.

Wartofsky L (2010). Increasing world incidence of thyroid cancer: increased detection or higher radiation exposure. Hormones, 9, 103-8.

Xhaard C, Ren Y, Clero E, et al (2014). Differentiated thyroid carcinoma risk factors in French polynesia. Asian Pac J Cancer Prev, 15, 2675. 